\title{
Effects of orienting task, spacing of repetitions, and list context on judgments of frequency
}

\author{
EDWARD J. ROWE and ROBERT J. ROSE \\ Memorial University of Newfoundland, St. John's, Newfoundland, Canada A1C 5S7
}

\begin{abstract}
Subjects were given an unexpected frequency judgment test following a list of words in which items were presented either two, three, and five times or three, five, and seven times, with a spacing of $0,2,16$, or 32 items between repetitions. During list presentation, they either rated the imagery value of each word or made continuous frequency estimates. Postlist frequency judgments of words presented three and five times were higher for the list containing words of Frequency 7 , and judgments were also higher following the imagery rating task. Continuous judgments were unaffected by the list context and showed different effects of spacing than postlist judgments. The results provide support for the operation of response bias factors in the frequency judgment task and are relevant to theoretical interpretations of the spacing effect.
\end{abstract}

One of the myriad pieces of information people have available about events in long-term memory is their frequency of occurrence. Average judgments of how often words occur in the English language (Shapiro, 1969) or in a specified situational context (Hintzman, 1969) closely reflect their true frequency of occurrence. The estimation of situational frequency has been examined in several recent experiments. Typically, subjects are shown a long list in which different words occur different numbers of times, followed immediately by a test trial in which the words are presented again, along with some new ones, for a numerical estimate. The basic finding in this task, which we will call a terminal judgment task, is a monotonic increase in average judgments as presentation frequency increases, with an "interaction" between true and judged frequencies such that low frequencies tend to be overestimated and high frequencies underestimated.

Several theoretical explanations of how frequency information is represented in memory have been reviewed by Hintzman (1976) and Howell (1973a). These include a trace strength hypothesis, whereby repetitions of an item serve to increase the strength of the trace of that item, a multiple-trace hypothesis, in which each repetition establishes its own independent trace, and a propositional coding view, in which frequency information is stored in an abstract propositional format. Hintzman's thorough evaluation of these hypotheses shows that trace strength is inadequate as an explanation of frequency representation, and while the available evidence does not clearly

This research was supported by Grant $A 8580$ from the National Research Council of Canada to E. J. Rowe. Madonna Tracey and Gillian Warrick assisted in the data collection and analysis. Send reprint requests to Edward J. Rowe, Department of Psychology, Memorial University of Newfoundland, St. John's, Newfoundland, Canada A1C 5S7. differentiate between the other two, the weight of it favors the multiple-trace hypothesis.

These hypotheses attempt to explain how a specific estimate is arrived at for each presented word. There have also been suggestions, however, that more general factors in addition to those responsible for individualitem assessment are involved in frequency judgments. Tversky and Kahnemann (1973) proposed that frequency estimates are biased by an availability heuristic, that is, the ease with which instances of a class of events come to mind. They gave people a list of names of famous males and not-so-famous females, or vice versa, and then asked them to recall whether there were more male or female names in the list. Most subjects incorrectly chose the class that contained the more famous names. Tversky and Kahneman argued that the judgment of relative frequency was preceded by a quick sample from memory of the male and female list names, to assess their relative availability. Since famous names are generally easier to recall, the sample will contain more names of famous people, which will lead to the conclusion that there were more famous names in the list. Rose and Rowe (1976) used the same idea to explain why words rated on a semantic dimension were given higher subsequent judgments than words processed for nonsemantic features. Semantic processing produces higher recall (Jenkins, 1974), so use of the availability heuristic would set a bias toward higher judgments on the frequency judgment test.

Other investigators have noted that subjects seem to develop a concept of the average frequency of occurrence of list items. Thus, the degree of overestimation of low frequencies and underestimation of high frequencies varies with the length of the retention interval (Underwood, Zimmerman, \& Freund, 1971) and the imagery value of words (Begg, 1974), but the variation in both cases occurs around a point which 
approximates the mean frequency of presentation. Judgments of individual words might be biased by this subjective average (Begg, 1974) such that words at extreme frequencies would show regression toward the mean. The subjective mean itself may be arrived at by a sampling procedure such as accompanies the availability heuristic.

One purpose of the present experiment was to strengthen the case for the influence of general biasing factors on judgments of situational frequency. Apart from frequency of presentation, there were three variables of interest. First, the words were presented in two different list contexts. If frequency judgments of individual items are biased by the availability or average subjective frequency of the items making up the list, then we expect that words presented the same number of times in two different lists will be given higher judgments in a list that also includes higher presentation frequencies than in one which includes only lower presentation frequencies. Accordingly, words were presented two, three, or five times in one list and three, five, or seven times in the other. Availability and the subjective mean are both higher in the second list than in the first, and this should lead to higher frequency estimates even for words occurring the same number of times in each list (i.e., with frequencies of three and five).

The subjects studied the presentation list under two types of processing tasks. They either rated each word on its ease of image arousal or made running (continuous) frequency judgments of the words as they were presented. Rose and Rowe (1976) have shown that orienting tasks which activate semantic processing of list items (rating of connotative strength and pleasantness) produce overestimations of presentation frequency, especially in the case of high-frequency words. An imagery rating task should insure semantic processing of each word, so the results here should be similar. In addition, the second group will allow us to compare performance on continuous and terminal frequency judgment tasks. Previous work (Begg, 1974; Begg \& Rowe, 1972) has shown that subjects' estimates on a continuous task conform much more closely to presented frequency than terminal judgments, even when the terminal test follows immediately after the continuous estimates. The over- and underestimation of low and high frequencies which characterizes terminal judgments is observed in a much reduced form, if at all, with continuous judgments.

There has been little theoretical speculation on how continuous judgments are made, other than a simple counting notion advanced by Begg and Rowe (1972). The direct comparison of continuous and terminal judgments could provide additional clues concerning the processes underlying each. For example, we can compare the effect of list context on continuous and terminal judgments. We have already outlined how this manipulation should affect terminal judgments, but its possible effect on continuous judgments is unclear. If the same sort of biases operate on continuous judgments as we suspect for a terminal task, parallel effects should be observed in both cases. On the other hand, the greater accuracy attached to continuous judgment suggests that they might be relatively unsusceptible to bias factors, and hence uninfluenced by list context.

The third variable of concern in the present experiment was spacing of repetitions; the words in the presentation list were repeated after $0,2,16$, or 32 intervening items. Here, more specific predictions concerning continuous vs. terminal judgments can be made. Rose and Rowe (1976) showed that terminal judgments increase with increased spacing, especially under instructions which prime semantic coding, and the increase is more pronounced with higher frequencies. To our knowledge, no one has studied the effect of spacing on continuous judgments. However, we know that the probability of recognizing an item that occurred previously in a long sequence shows a monotonic decrease with increases in spacing (Shepard \& Teghtsoonian, 1961). If recognition of a repetition as such is a prerequisite for continuous frequency judgment, as it surely must be, then we might expect to find a similar decreasing function here, which is the opposite of what happens with terminal judgments. Thus, the spacing variable might provide another functional distinction between continuous and terminal judgments.

To summarize, the subjects saw a list of words in which repetitions occurred either two, three, and five times or three, five, and seven times, separated by a spacing of $0,2,16$, or 32 other items. In going through the list, they either made continuous judgments of the frequency of the words or rated them on an imagery scale, both of which were followed by the same terminal judgment test.

\section{METHOD}

\section{Materials and Design}

The two lists were constructed from a pool of common words (A or AA by the Thorndike-Lorge count) which had one or two syllables and five or six letters each. In one list, different words were repeated two, three, or five times (call this List 235), while in the other the frequencies were three, five, and seven (List 357). Sixteen different words occurred at each frequency, and 51 additional words in each list occurred only once. Once-presented words occupied the first four and last four list positions as primacy and recency buffers. List 235 thus had a total of 211 items and List 357,291 . We consider this confounding of list type with list length to be unimportant, since Howell (1973b) has found equivalent frequency judgments for words in lists which varied between 46 and 368 items in length.

Repetitions of words were separated by $0,2,16$, or 32 other items. Four of the 16 words at each frequency greater than one were represented at each degree of spacing. An effort 
was made to have the four words of each frequency by spacing combination distributed equally throughout the entire list. Where equal distribution was not possible (with higher frequencies and longer spacing intervals), it was approximated.

Eight versions of each list were drawn up in order to increase the word sample occurring in each frequency by spacing condition. Thus, across all eight versions of the list, the words were rotated through all 12 conditions. This was done in such a way that each word appeared exactly twice at each level of spacing and in approximately equal proportion at each level of frequency.

The fourth factor in the design was the type of orienting task given. Half of the subjects made written judgments of the frequency of occurrence of each word as it appeared in the list, writing 1 for a first occurrence, 2 for a second, and so on. The others made 7-point ratings of how easily they could generate an image for each word. The complete design was therefore a 2 (task) by 2 (type of list) by 3 (frequency) by 4 (spacing) factorial, with repeated measures on the last two factors. Thirty-two subjects were assigned to each of the between-subjects conditions.

\section{Procedure}

The words were typed on index cards for presentation to the subjects, who went through the assigned deck of cards at their own rate. Four subjects in each study condition received one of the eight versions of the appropriate list. Two of these subjects went through the deck in one direction and two in the reverse direction. A practice deck of 12 items preceded presentation of the experimental list. No mention was made of the fact that a frequency judgment test would be given following list presentation. Some of the subjects were tested individually, but most were tested in squads of six or less. When all subjects had finished going through the deck of cards, they were given the frequency judgment test sheet with instructions to write beside each word the number of times it had appeared in the deck. The 80 items on the test sheet, arranged in four columns of 20 items each, consisted of the four words from each frequency by spacing combination, a random sample of 16 of the 51 once-presented words, and 16 words which had not appeared in the presentation sequence. An equal number of these three types of test items occurred on each half of the test sheet.
Subjects

Most of the subjects were undergraduates at Memorial University. The others were recruited from a pool of high school students on file at the Canada Manpower office in St. John's. All subjects were paid.

\section{RESULTS}

The results will be presented in three sections, dealing with performance on the terminal test, performance on the continuous test, and comparisons between the two.

\section{Terminal Judgments}

The mean frequency judgment assigned to the four words in each spacing by frequency combination is shown as a function of the four independent variables of the experiment in Figure 1. The main findings are as follows: The words were given higher mean judgments following the imagery rating task (5.37) than following the continuous judgment task (3.43), but the effects of the other three factors were very similar in each case. Thus, as expected, judgments increased systematically with increases in presentation frequency for each of the two list conditions. The spacing effect was also typical, with generally higher judgments accompanying longer spacing intervals in the form of a negatively accelerated function. The function shows both an increased initial slope and a higher asymptote for higher levels of presented frequency, as found by Rose and Rowe (1976). Finally, those frequencies (three and five) which were common to the two lists were given higher judgments when they occurred in List 357 than in List 235.

The data of Figure 1 were analyzed by a series of analyses of variance. In all statistical results reported

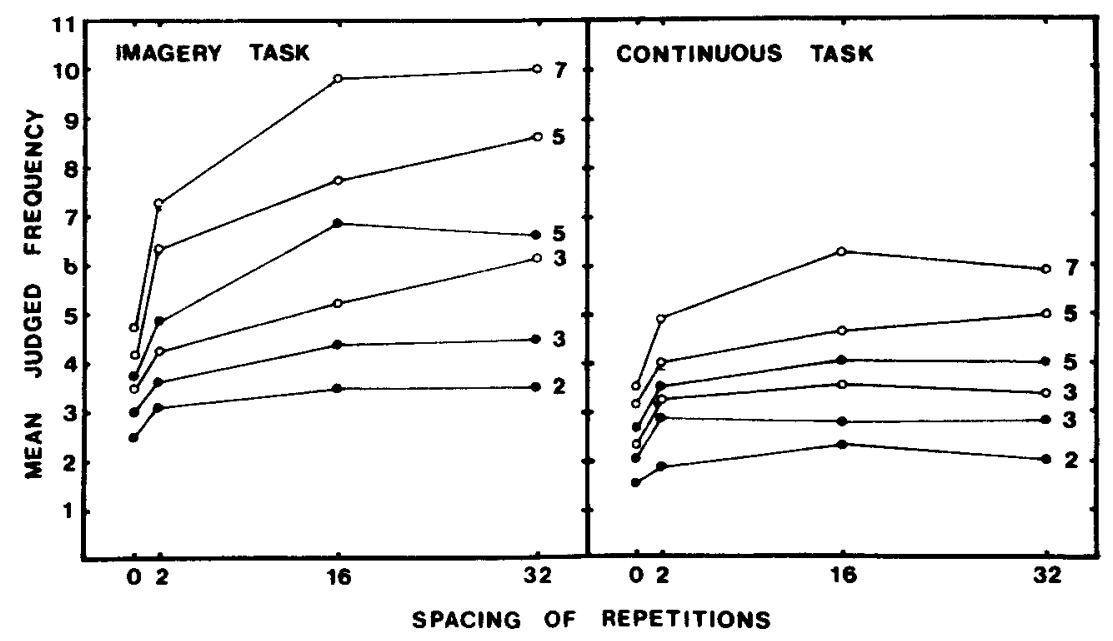

Figure 1. Mean frequency judgment on the terminal test as a function of task, list, and spacing of repetition. List 235 is represented by closed circles and List 357 by open circles. Presented frequency is given next to each curve. 
here and throughout the remainder of this paper, the probability of rejecting the null hypothesis in error is .05. The first analysis compared judgments of Frequencies 3 and 5 , which are common to all conditions, as a function of task, list, and spacing. The results confirmed our initial impressions. The imagery task produced higher judgments than the continuous task $[\mathrm{F}(1,124)=40.3, \mathrm{MSe}=22.6]$, and there were significant main effects of presentation frequency $[\mathrm{F}(1,124)=234, \mathrm{MSe}=2.18]$, and spacing $[F(3,373)=100, M S e=2.25]$. More important for purposes of the present report was the finding that significantly higher judgments were given in the context of List 357 than List $235 \quad[F(1,124)=6.72$, $\mathrm{MSe}=22.7]$, which is direct support for a biasing effect of list context. Several of the two-way interactions were significant. The wider separation of frequencies in judgments following the imagery task was reflected in a Task by Frequency interaction $[F(1,124)=16.67$, $\mathrm{MSe}=2.18]$, and the enhancement of the spacing effect by the imagery task produced an interaction of Task by Spacing $[\mathrm{F}(3,372)=14.5, \mathrm{MSe}=2.25]$. The Frequency by Spacing interaction $[F(3,372)=9.88$, $\mathrm{MSe}=2.11]$ and the interaction of List by Spacing $[\mathrm{F}(3,372)=3.77, \mathrm{MSe}=2.25]$ can be similarly interpreted as an enhancement of the spacing effect by higher presentation frequencies and List 357 , respectively. None of the remaining interactions was significant.

The effects of presentation frequency and spacing were further examined by separate two-way analyses of variance on the data of each group of subjects receiving one of the four list by task conditions. These analyses included frequencies of two and seven, respectively, which were omitted from the main analysis. The results were straightforward: Significant main effects of frequency and spacing and a significant Frequency by Spacing interaction were found in each case.

We also measured the degree of error, or conversely, the accuracy attached to the subjects' frequency estimates as represented in Figure 1. For each individual word, the unsigned difference between the subject's estimate and the true frequency of presentation was calculated (Rose \& Rowe, 1976). These deviation scores reflect the accuracy with which subjects recall the frequency of each word. The measure lumps together overestimates and underestimates, so we lose information about the direction of judgment error, but the data are useful as an adjunct to average estimates, in which the accuracy of individual judgments is obscured. The major findings, confirmed by analysis of variance, were larger deviations with higher presentation frequency, larger deviations for List 357 than List 235, and a differential spacing effect for the two orienting tasks, such that the average deviation increased with longer spacing intervals for the imagery task, but decreased for the continuous task. The reason for this interaction is apparent from Figure 1. Subjects tended to overestimate frequencies at longer spacing intervals following the imagery rating task, as compared with a tendency to underestimate at shorter intervals following the continuous task.

Flexser and Bower (1975) have developed an index of frequency judgment performance which measures the degree of discriminability among levels of presented frequency. This discrimination coefficient is the correlation between presented frequency and judged frequency for a given set of experimental conditions. It provides a measure of discriminability which is free from the influence of bias factors that indiscriminately raise or lower all levels of presented frequency by a multiplicative constant. As long as the proportionality between judged frequencies corresponding to different levels of presented frequency is unaffected, the discrimination coefficient remains unchanged. Biases which might selectively affect specific parts of the range of judged values-extreme frequencies, for example-are not controlled for by this measure.

We calculated discrimination coefficients (Pearson's r) for the data of Figure 1. A coefficient was calculated for each subject at each level of spacing in each of the four task by list conditions, and normalized by the $\mathrm{z}_{\mathrm{r}}$ transformation for analysis. The mean coefficients, transformed back into $r$ scores, are given in Table 1 . A 2 (task) by 2 (list) by 4 (spacing) analysis of variance was carried out, which produced a significant effect only for the spacing factor. As indicated in Table 1 , and confirmed by Newman-Keuls comparisons, discriminability among the three levels of presented frequency was lower for massed repetitions than for those presented at nonzero spacings, which did not differ among themselves. The lower judgments given massed repetitions at higher frequencies seem to reflect a true memory deficit in the sense that subjects were poorer in discriminating among the three levels of presented frequency. On the other hand, the failure to find an effect of either orienting task or list context implies that the differences in average judgments

Table 1

Mean Discrimination Coefficients for Terminal Frequency Judgments as a Function of Spacing and Instructions for Lists 235 and 357

\begin{tabular}{lcccc} 
& \multicolumn{4}{c}{ Spacing } \\
\cline { 2 - 5 } Type of Task & 0 & 2 & 16 & 32 \\
\hline Continuous Judgment & & & \\
List 235 & .80 & .90 & .93 & .94 \\
List 357 & .63 & .87 & .93 & .91 \\
Imagery Rating & & & & \\
List 235 & .76 & .90 & .96 & .96 \\
List 357 & .74 & .94 & .95 & .92 \\
Mean & .74 & .91 & .95 & .94 \\
\hline
\end{tabular}


Table 2

Mean Frequency Judgments on the Terminal Test for Presentation Frequencies of Zero and One

\begin{tabular}{crrrr} 
& \multicolumn{4}{c}{ Task } \\
\cline { 2 - 5 } $\begin{array}{c}\text { Presen- } \\
\text { tation }\end{array}$ & \multicolumn{2}{c}{ Continuous Judgment } & & \multicolumn{2}{c}{ Imagery Rating } \\
\cline { 2 - 5 } \cline { 5 - 6 } Frequency & List 235 & List 357 & List 235 & List 357 \\
\hline 0 & .47 & .50 & .31 & .30 \\
1 & 1.30 & 1.49 & 1.79 & 2.03 \\
\hline
\end{tabular}

produced by these variables are the result of response bias.

So far, we have not considered judgments of words presented with frequencies of zero and one. These are shown in Table 2, and can be dealt with summarily. Judgments of zero words did not vary with different task and list conditions. There was some tendency to give higher estimates to zero words following the continuous task, but a task by list analysis of variance showed no significant effects. Once-presented words were given higher judgments following the imagery task $[\mathrm{F}(1,124)=9.74, \quad \mathrm{MSe}=.877]$, but were not affected by the type of list, nor was there an interaction between the two.

\section{Continuous Judgments}

The mean judgments assigned to words on the continuous test are shown as a function of frequency, spacing, and list type in Figure 2. These judgments deviate very little from the actual frequencies of presentation, and except for the higher frequencies in List 357 , are little affected by the spacing variable. There is also little variation between lists. Three analyses were carried out on these data, excluding judgments of words that occurred only once. The first two were separate analyses of variance for each list, with frequency and spacing as factors. Frequency was of course a significant source of variance both for List $235 \quad[F(3,93)=648, \quad M S e=.261]$ and List 357 $[F(5,155)=361, M S e=.996]$. The effect of spacing was significant only for List $357 \quad[F(3,93)=3.85$, $\mathrm{MSe}=2.74]$, as was the Frequency by Spacing interaction $[\mathrm{F}(15,465)=3.34, \mathrm{MSe}=.446]$. Frequency judgments showed a tendency to decrease with increased spacing for words presented five times or more. Since judgments of the second, third, and fourth repetitions do not drop off with increased spacing, it must be the later repetitions of more frequent items that suffer the effects of longer spacing intervals. The spacing effect in this case seems due not so much to what happens during the interval between each repetition as to the cumulative effect of several successive spacing intervals. This is a different picture than the one shown by terminal judgments, where the influence of spacing is observed with few as well as many repetitions. It also differs from results obtained in continuous recognition memory, where the drop-off occurs for twice-presented words over the same range of spacing intervals used here (Shepard \& Teghtsoonian, 1961).

The third analysis of the continuous judgment data examined the effect of list context. As before, this was done by comparing estimates of those items repeated the same number of times in both lists, in this case two, three, four, and five. Frequency $[\mathrm{F}(3,186)=1,057$, $\mathrm{MSe}=.322]$ and spacing $[\mathrm{F}(3,186)=3.40, \mathrm{MSe}=.972]$ were both significant, as was their interaction $[F(9,558)=5.23, \mathrm{MSe}=.195]$. In contrast to what was found with terminal judgments, list context had no effect, nor did it interact with the other two variables.

\section{Continuous vs. Terminal Judgments}

The final set of analyses compared judgments on the continuous and terminal tests for those subjects who received both. The mean judgment assigned to the final repetition of the words of each frequency on the continuous test was compared with judgments of the same items on the terminal test. The data are shown in Figure 3. The curves for terminal judgments are the same as those shown in Figure 1, and the curves

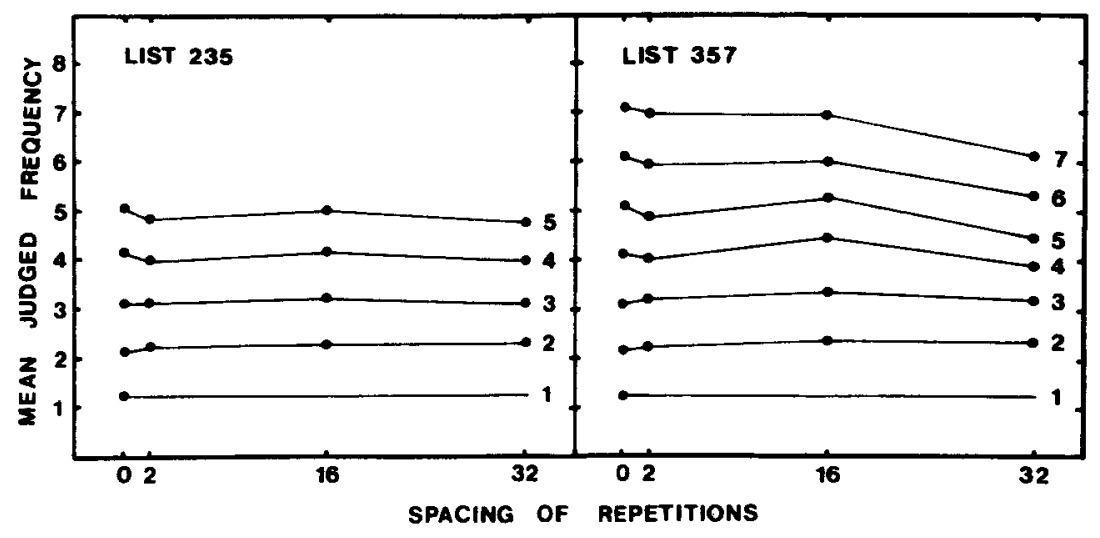

Figure 2. Mean frequency judgment on the continuous test as a function of list type and spacing of repetitions. Presented frequency is given next to each curve. 


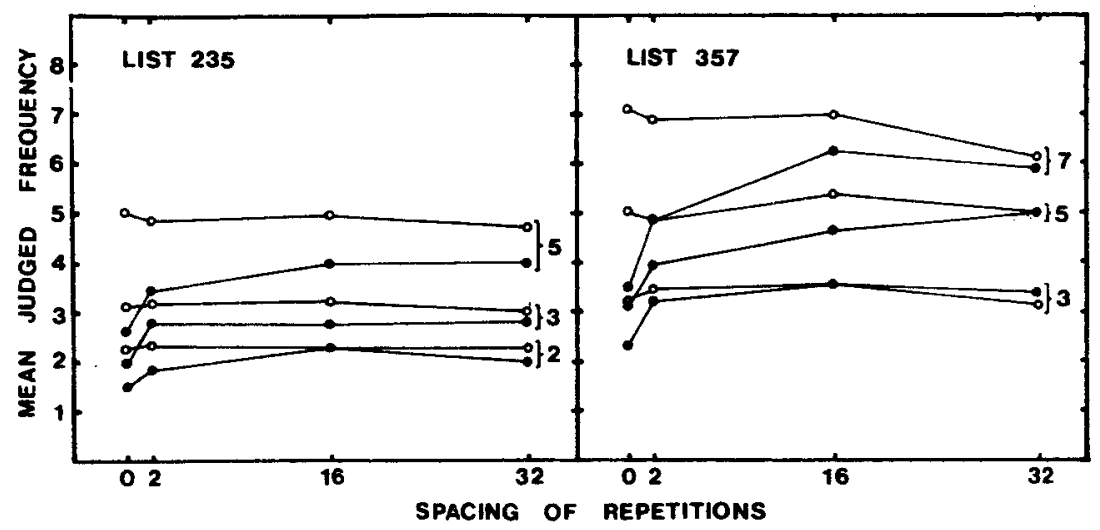

Figure 3. Mean frequency judgment on the continuous and terminal tests as a function of list type and spacing of repetitions. Terminal judgments are represented by closed circles and continuous judgments by open circles. Presented frequency is given next to each curve.

for continuous judgments of words of Frequency 5 in List 235 and words of Frequency 7 in List 357 are identical to the corresponding curves of Figure 2. The other continuous judgment functions differ slightly from those of Figure 2, since they are based on only the final repetition at each frequency, whereas the Figure 2 data reflect the average judgment of all repetitions at a given frequency. Figure 3 shows a systematic relation between continuous and terminal judgments of the same words. Generally speaking, the spacing function observed for terminal judgments at each presentation frequency rises to asymptote with the corresponding function for continuous judgments.

Separate analyses of variance were done for each list, with an identical pattern of results in each instance. Continuous judgments were higher than terminal judgments overall $[F(1,31) \leqslant 22.0]$, and the main effects of frequency $[F(2,62) \leqslant 173]$ and spacing $[F(3,93) \leqslant 13.4\rceil$ were significant. The type of judgment (continuous or terminal) interacted with frequency $[F(2,62) \leqslant 32.1]$, reflecting the larger separation between the two types of judgments at higher frequencies, and with spacing $[F(3,93) \leqslant 26.3]$, reflecting the larger separation between the two at shorter spacing intervals. A significant three-way interaction of Type of Judgment by Frequency by Spacing $[F(6,186) \leqslant 4.88]$ showed that the difference between continuous and terminal judgments was a joint function of both presentation frequency and degree of spacing, as indicated in Figure 3. The interaction between the two spacing functions was largest at higher frequencies.

\section{DISCUSSION}

The main findings may be summarized as follows: Terminal frequency judgments were higher, on the average, and the accuracy of such judgments was lower for words given imagery ratings than for words given continuous frequency estimates on the study list. Higher judgments and lower accuracy also resulted from presentation of words of the same frequency in the context of List 357 compared to List 235. Typical negatively accelerated functions were found for the spacing variable when average judgments on the terminal test constituted the performance measure, the rate of increase and final asymptote being higher for the imagery task and higher levels of presented frequency. Average continuous judgments were unaffected by list context and, compared to terminal judgments, affected in a different way by the spacing variable. Spacing had an effect only at higher frequencies of presentation (greater than four), where judgments decreased with increased spacing. A comparison of the final judgments of words on the continuous test with terminal judgments of the same words showed that the drop-off in average estimates on the terminal test was greater for shorter spacing intervals and higher presentation frequencies.

Several sources of evidence for the influence of bias factors in terminal frequency judgments were found. As expected, estimates of words which occurred the same number of times in the different list contexts were influenced by the values of the accompanying frequencies. If the process of frequency judgment involved only the independent assessment of some aspect of the memory representation of each word, then words of equal frequency should, on the average, have been given equivalent estimates regardless of how often other words in the list were presented. Since the results reflected the distribution of accompanying frequencies, we conclude that the subjects' frequency estimates were adjusted to take into account the average frequency, or perhaps the range of frequencies, of the presentation list.

Additional evidence for bias effects comes from the analysis of discrimination coefficients. The results here corroborated a bias interpretation of the list context 
effect in showing that the effect obtained only when raw frequency estimates were considered. When the relative discriminability of frequencies was assessed independent of their absolute values, no effect of list context was found. Thus, although changing the list context introduced a bias which altered the overall level of frequency estimates, the relative difference between different frequencies was still maintained. The same was true of the instructional effect. The imagery rating task produced an overestimation of words at all frequency levels, as found by Rose and Rowe (1976) for other semantic tasks, while judgments following the continuous task were closer to the actual presentation frequencies. Discrimination coefficients, however, were the same for the two task conditions, suggesting that the inflated judgments given by the imagery rating group reflect a bias toward overestimation. This bias can be interpreted in terms of Tversky and Kahnemann's (1973) availability heuristic, if we assume that imagery increased the availability of the list items relative to making continuous estimates.

In contrast to terminal judgments, continuous judgments seemed to be relatively unaffected by bias, since they showed no effect of the list context manipulation. What this suggests is that those factors which tend to bias terminal judgments of specific items are set up after the study list has been presented, prior to the beginning of the test list or perhaps while the first few judgments are being made. At this point factors such as the perceived availability of list items, their average frequency, and the range of frequencies presented establish a general cognitive context for making frequency estimates of the individual test items, a context which will bias these individual estimates to bring them in line with the subject's overall impression of the frequency structure of the presentation list. Thus the judgment process may consist of two subcomponents, the setting of the bias factors followed by a more detailed estimation of each individual item. To date, theoretical explanations have focused on the second of these components (cf. Hintzman, 1976) to the relative neglect of the first. The present results suggest that the bias component be taken into account by any theory attempting a complete explanation of the frequency estimation process.

The above comments notwithstanding, the difference between continuous and terminal judgments of the same words cannot be explained solely in terms of the presence or absence of bias factors. For massed repetitions, at least, there appears to be a genuine memory loss between the final presentation of the word on the study list and its occurrence on the terminal test. Subjects showed almost perfect accuracy in continuous judgments of massed items, but these same items then received the lowest estimates on the terminal test. That this difference is not merely one of response bias was suggested by the analysis of discrimination coefficients, which were lower for massed than for spaced repetitions. Given that discrimination coefficients are bias free, this loss of discriminability among different levels of frequency for massed items represents a real memory deficit.

Finally, the results are relevant to theoretical interpretations of the spacing effect itself as manifested in terminal frequency judgments. Hintzman (1976) has distinguished between encoding variability and deficient processing theories of the spacing effect. Encoding variability theories hypothesize that spaced repetitions are more likely to receive differential coding than massed items, making them more retrievable, while deficient processing theories view the spacing effect as the result of poorer processing of massed items. The comparison of continuous and terminal judgments in the present experiment clearly supports the latter idea. Taking final continuous judgments as the baseline against which terminal judgments can be compared, it is obvious that what happens on the terminal test is a lowering of judgments for massed repetitions, rather than an enhancement of judgments for spaced items. This finding was also supported by the discrimination coefficient analysis, which showed a loss of discriminability among presented frequencies only for massed items between the continuous and terminal tests. For the frequency judgment task, then, theories of the deficient processing variety are preferable to encoding variability as an explanation of the spacing effect.

\section{REFERENCES}

BEGG, I. Estimation of word frequency in continuous and discrete tasks. Journal of Experimental Psychology, 1974, 102. 1046-1052.

BeGG, I., \& Rowe, E. J. Continuous judgments of word frequency and familiarity. Journal of Experimental Psychology, 1972. 95. 48-54

FleXser, A. J.. \& Bower. G. H. Further evidence regarding instructional effects on frequency judgments. Bulletin of the Psychonomic Society. 1975, 6. 321-324.

Hintzman, D. L. Apparent frequency as a function of frequency and the spacing of repetitions. Journal of Experimental Psvchology. 1969, 80, 139-145.

Hintzman, D. L. Repetition and memory, In G. H. Bower (Ed.). The psychology of learning and motivation (Vol. 10). New York: Academic Press, 1976.

HowelL. W. C. Representation of frequency in memory. Psychological Bulletin, 1973, 80, 44-53. (a)

Howell, W. C. Storage of events and event frequencies: A comparison of two paradigms in memory. Journal of Experimental Psychology. 1973, 98, 260-263. (b)

JENkINS. J. J. Can we have a theory of meaningful memory? In R. L. Solso (Ed.). Theories in cognitive psychology: The Lovola simposium. Potomac, Md: Erlbaum. 1974.

Rose, R. J.. \& Rowe. E. J. Effects of orienting task and spacing of repetitions on trequency judgments. Journal of Experimental Psychology: Human Learning and Memory. 1976. 2. 142-152.

SHAPIRO, B. J. The subjective estimate of relative word frequency. Journal of Verbal Learning and Verbal Behavior. $1969,8.148-251$. 
Shepard, R. N., \& Teghtsoonian, M. Retention of information under conditions approaching a steady state. Journal of Experimental Psychology, 1961, 62, 302-309.

TVersky, A., \& KahnemanN, D. Availability: A heuristic for judging frequency and probability. Cognitive Psychology, 1973, 5, 207-232.

UNDeRWOOD, B. J., Zimmerman, J., \& Freund, J. S.
Retention of frequency information with observations on recognition and recall. Journal of Experimental Psychology, 1971, 87, 149-162.

(Received for publication May 10, 1977; accepted June 10, 1977.) 